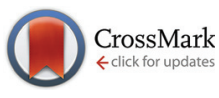

Cite this: Dalton Trans., 2015, 44 12535

Received 15th May 2015,

Accepted 3rd June 2015

DOI: $10.1039 / \mathrm{c} 5 \mathrm{dt} 01839 \mathrm{k}$

www.rsc.org/dalton

\title{
Modeling the magnetic properties of lanthanide complexes: relationship of the REC parameters with Pauling electronegativity and coordination number†
}

\author{
José J. Baldoví, Alejandro Gaita-Ariño* and Eugenio Coronado
}

\begin{abstract}
In a previous study, we introduced the Radial Effective Charge (REC) model to study the magnetic properties of lanthanide single ion magnets. Now, we perform an empirical determination of the effective charges $\left(Z_{i}\right)$ and radial displacements $\left(D_{r}\right)$ of this model using spectroscopic data. This systematic study allows us to relate $D_{\mathrm{r}}$ and $Z_{i}$ with chemical factors such as the coordination number and the electronegativities of the metal and the donor atoms. This strategy is being used to drastically reduce the number of free parameters in the modeling of the magnetic and spectroscopic properties of f-element complexes.
\end{abstract}

The unique spectroscopic and magnetic properties of lanthanide ions have excited researchers for decades. ${ }^{1}$ These systems present more complex and intricate spectra than those of transition metals, which are characterized by a few broad bands. In this context, crystal-field theory is essential to analyze and simulate the energy level schemes of f-element complexes, ${ }^{2}$ providing an accuracy level of about $10 \mathrm{~cm}^{-1} \cdot{ }^{3-5}$ The determination of the crystal field parameters (CFPs) is a fundamental problem in materials used in solid state physics. The CFPs can be deduced from a direct fit of UV/visible spectroscopic experimental data. ${ }^{6}$ Also, information about the lower lying magnetic levels can be obtained from the far infrared (FIR) ${ }^{7}$ or from inelastic neutron scattering (INS) spectra. ${ }^{8}$ In any case, the fitting procedures need a trial set of non-zero CFPs, while the rest of the CFPs are set to zero because of the idealized symmetry of the complex, this strategy is valid for simple salts. However, when dealing with molecular systems, this strategy requires the determination of a large number of CFPs, resulting in overparametrization. In these cases, the idealization of the chemical structure is often unreasonable and this constitutes an open problem. Since such procedures involve a function of many variables which have to be fitted, starting from a good trial set of CFPs is necessary in order to reach good

Instituto de Ciencia Molecular (ICMol), Universidad de Valencia, C/Catedrático José Beltrán 2, E-46980 Paterna, Spain.E-mail: gaita@uv.es

$\dagger$ Electronic supplementary information (ESI) available. See DOI: 10.1039/ c5dt01839k convergence with minimum error. ${ }^{9}$ This initial set of CFPs can be calculated from the real structure of the compounds using Complete Active Space $a b$ initio calculations ${ }^{10}$ or, more inexpensively, by a simple point-charge electrostatic model (PCEM). ${ }^{11}$ This latter approach, due to its inherent limitations, was subsequently improved by several semiempirical models. ${ }^{12-17}$

In this context, we developed the Radial Effective Charge (REC) model in 2012, modeling the effect of the ligating atom through an effective charge situated along the lanthanoiddonor atom axis. ${ }^{18}$ The REC model has become very useful for quick predictions of the magnetic properties and for obtaining an initial set of CFPs, and has been applied with success to a large variety of f-element complexes with interesting optical ${ }^{19}$ and magnetic properties. ${ }^{20}$ This approximation also needs parameterization to provide a more realistic description of the properties. By taking into account covalency, it relies on two parameters, namely the radial displacement $\left(D_{\mathrm{r}}\right)$ and the effective charge $\left(Z_{i}\right)$, that are obtained from a direct fit of the experimental spectroscopic and/or magnetic properties. The predictive power of this approach was recently demonstrated in a series of isostructural polyoxometalates, ${ }^{21}$ suggesting the idea of the utility of building a general library for the inexpensive rationalization of the properties of rare earth compounds. Nevertheless, it is not clear whether these effective parameters really are transferrable between different complexes, independently of the coordination number.

In this communication, we perform a systematic application of the REC model to a series of later lanthanide ions in crystal hosts. We have chosen four families that present a lanthanoid ion coordinated by halides: fluorides and chlorides, as model systems. The spectroscopic studies ${ }^{22}$ and the determination of CFPs that have been carried out on these systems permit us to obtain reliable $D_{\mathrm{r}}$ and $Z_{i}$ parameters. The general formula of the studied lanthanide ions in crystal hosts are: $\mathrm{Cs}_{2} \mathrm{NaYCl}_{6}: \mathrm{Ln}^{3+}$ (Cl6), $\mathrm{LaCl}_{3}: \mathrm{Ln}^{3+}$ (Cl9) and $\mathrm{LiYF}_{4}: \mathrm{Ln}^{3+}$ (F8), where $\mathrm{Ln}=\mathrm{Tb}$, Dy, Ho, Er and Tm, and the isostructural family, $\mathrm{Cs}_{2} \mathrm{NaYF}_{6}: \mathrm{Ln}^{3+}$ (F6), in which the data of Ho and $\mathrm{Er}$ compounds are used in the fit. We extracted the REC parameters using two different procedures in the modified versions 
of the SIMPRE package. ${ }^{23}$ The first one consists of the fitting of the ground multiplet energy level scheme for each lanthanoid. For the description of the whole series, a second step is the collective fit: we obtain the best combination of $D_{\mathrm{r}}$ and $Z_{i}$ with minimum mean squared error. In addition, the phenomenological CFPs of each lanthanide complex involved in this study are directly fitted. The results of the calculations concerning CFPs and energy levels are compared with the phenomenological CFPs and spectroscopic energy levels and reported in the ESI. $\dagger$

The results of every individual fit, taken together with the collective fits for each family, reveal at first glance some trends that can be related to both the Pauling electronegativities of the ligands and the coordination numbers (see Fig. 1). For instance, for a given coordination number $(\mathrm{CN})$, e.g. $\mathrm{CN}=6, D_{\mathrm{r}}$ is higher for the ligand with a lower electronegativity $\left(E_{\mathrm{Cl}}<E_{\mathrm{F}}\right)$. Additionally, for a given donor atom, $D_{\mathrm{r}}$ is higher for a higher coordination number. This is verified for both $\mathrm{F}^{-}$and $\mathrm{Cl}^{-}$. This means that as the coordination number increases, the effective charge gets closer to the lanthanoid. On the other hand, a more electronegative donor atom will retain the effective charge a bit longer with respect to the rare earth ion, resulting in a less covalent coordination bond.

Previous efforts in this field (see for example ref. 17) attempted to find relatively simple relationships between some kind of effective charge parameters and chemical concepts such as the coordination number or the electronegativity. In the light of the present data, a careful analysis of the collective fit numerical data results in the following approximate relationship among the radial displacement $\left(D_{\mathrm{r}}\right)$, the coordination number $(\mathrm{CN})$, the valence of the metal $\left(V_{\mathrm{M}}\right)$ and the Pauling electronegativities of the ligand and the metal $\left(E_{\mathrm{L}}\right.$ and $E_{\mathrm{M}}$, respectively):

$$
D_{\mathrm{r}} \approx\left(\frac{\mathrm{CN}}{V_{\mathrm{M}}}\right) \frac{1}{E_{\mathrm{M}}\left(E_{\mathrm{L}}-E_{\mathrm{M}}\right)}
$$

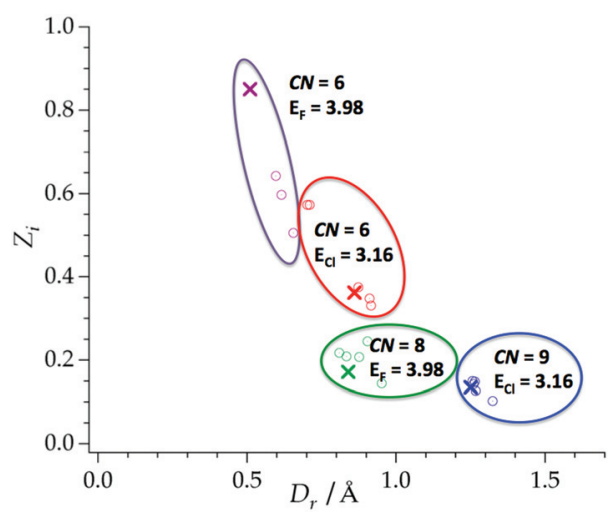

Fig. $1 D_{\mathrm{r}}$ and $Z_{i}$ parameters obtained for each CFP individual fit (open circles), collective fitting of the energy levels (cross) for the families $\mathrm{Cl6}$ (red), F8 (green), Cl9 (blue) and F6 (purple).
We arrived at this expression by taking advantage of the fact that $E_{\mathrm{L}}-E_{\mathrm{M}}$ is inversely related to the degree of covalency of a chemical bond. Table 1 shows a comparison between the approximation given in eqn (1) and the collective fit of the ground multiplet energy level scheme of each family. Thus the calculated energy levels, which are from the standard and recommended procedure of the REC model, are compared with the experiment in ESI Fig. 6-9. $\dagger$ The diagonally calculated CFPs are compared with the phenomenological ones, which are taken from the experiment, in ESI Fig. 10-12. $\dagger$ Also, in Table 1 , the averages of the $D_{\mathrm{r}}$ obtained within the individual fits of the four families, based on ground multiplet energy levels and crystal field parameters, are reported.

As can be observed, the agreement is reasonable for the four families. Note that for the F6 family, marked with $\left(^{*}\right)$ in Table 1, the collective fit uses only the data of $\mathrm{Ho}^{3+}$ and $\mathrm{Er}^{3+}$, and this value is expected to change if we take into account more derivatives.

Regarding the obtained effective charges of these complexes, an inversely proportional relationship with respect to the $D_{\mathrm{r}}$ values may be observed in both fluoride and chloridebased families (Fig. 2 and ESI Fig. $5 \dagger$ ). This is indeed a general trend in all the systems fitted using this model: as long as the effective charge gets closer to the lanthanoid ion, its value decreases (ESI Fig. 1-4†). The reason for this is obvious: if a solution is already in the right parameter region, pushing the charges closer to the metals and simultaneously increasing their effective values will produce a completely incorrect total CF splitting. In Fig. 2, we have plotted the individual $D_{\mathrm{r}}$ and $Z_{i}$ values obtained when fitting the ground multiplet CF splitting, the ones obtained when fitting the CFPs directly and the lowest relative error combination that describes the ground multiplet energy levels of the whole series. As an approximation, the following relationship can be assumed:

$$
f_{\mathrm{X}, \mathrm{CN}}=D_{\mathrm{r}} Z_{i}
$$

where $f$ is a factor which depends on the coordination number and on the coordinated atom $(\mathrm{X})$.

The resulting values of $f_{\mathrm{X}, \mathrm{CN}}$ are calculated using the REC parameters of the collective fit. The values are: $f_{\mathrm{F}, 6}(\mathrm{CN}=6)=$ $0.434, f_{\mathrm{F}, 8}(\mathrm{CN}=8)=0.144, f_{\mathrm{Cl}, 6}(\mathrm{CN}=6)=0.311$ and $f_{\mathrm{Cl}, 8}(\mathrm{CN}=$ $9)=0.169$. For the two families coordinated by chlorides, the relationship $Z_{i}=f_{\mathrm{Cl}} / D_{\mathrm{r}}$ is calculated keeping the averaged $f_{\mathrm{Cl}}=$

Table $1 D_{\mathrm{r}}$ estimated either from eqn (1) by a collective fit of each family or as an average of the individual fits of the energy levels and of the CFPs. $(*)$ : see the text

\begin{tabular}{lllll}
\hline & $D_{\mathrm{r}}(\AA)$ & & & \\
\cline { 2 - 5 } & Eqn (1) & Collective fit & Fit energy levels (ave.) & Fit CFPs (ave.) \\
\hline F6 & 0.6 & $0.51\left(^{*}\right)$ & $0.57\left(^{*}\right)$ & $0.57\left(^{*}\right)$ \\
F8 & 0.8 & 0.84 & 0.81 & 0.88 \\
C16 & 0.85 & 0.86 & 0.90 & 0.85 \\
C19 & 1.28 & 1.25 & 1.24 & 1.27
\end{tabular}




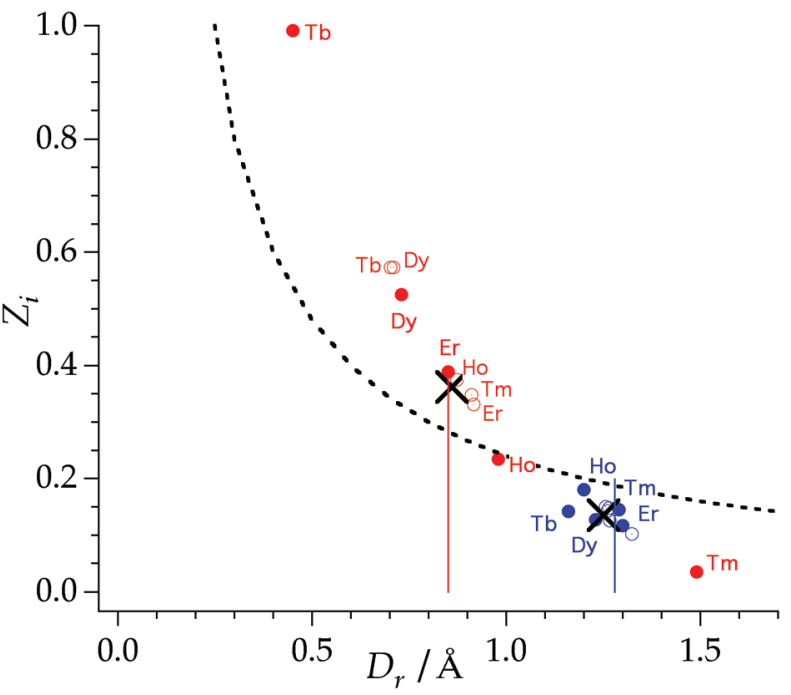

Fig. 2 Radial displacement $\left(D_{\mathrm{r}}\right)$ and effective charge $\left(Z_{i}\right)$ values obtained by fitting the ground multiplet energy levels (circles) and crystal field parameters (open circles); $\mathrm{Cs}_{2} \mathrm{NaYCl}_{6}: \mathrm{Ln}^{3+}$ (red) and $\mathrm{LaCl}_{3}$ : $\mathrm{Ln}^{3+}$ (blue). Collective fit (black cross). Dashed line: function $Z_{i}=f_{\mathrm{Cl}} / D_{\mathrm{r}}$; vertical lines: $D_{\mathrm{r}}$ from eqn (1).

$\left(f_{\mathrm{Cl}, 6}+f_{\mathrm{Cl}, 9}\right) / 2$ constant. This function is represented by the dashed black line in Fig. 2. For F6 and F8, the function $Z_{i}=$ $f_{\mathrm{F}} / D_{\mathrm{r}}$ is plotted in ESI Fig. $5 . \dagger$

Relationships (1) and (2) presented in this communication allow an initial estimation of the CFPs, energy levels, magnetic and optical properties for homoleptic later lanthanide complexes. They can be especially useful to obtain an initial trial of CFPs for spectroscopic studies in f-element complexes using the full Hamiltonian. Also, the REC parameters obtained can be used as initial values for $D_{\mathrm{r}}$ and $Z_{i}$ in the fitting procedures when modeling the properties of this kind of system in an inexpensive way.

For perspective, let us compare the approximations presented in eqn (1) and (2) with previous efforts towards this direction. There are several similarities between the estimation of the effective charge in ref. 17, where a modified pointcharge model is presented, and the present one, namely, the effective charge:

(a) is directly proportional to the valence of the metal;

(b) is inversely proportional to the number of ligands;

(c) becomes zero as the electronegativity of the ligand approaches the electronegativity of the metal; and

(d) can be written as a negative number plus a term that depends linearly on the electronegativity of the ligand.

Furthermore, in a recent work by Tanner et al. ${ }^{24}$ (c) is exactly true and (d) is approximately true if one considers small differences in electronegativity, as one then can look at the linear approximation of a negative Gaussian function. As ref. 24 considers a constant radius depending only on the metal ion, it also supports the proportionality between $D_{\mathrm{r}}$ and $\mathrm{CN}$ : crystallographic radii increase with the coordination number, thus a rising radial displacement is needed to compensate for this if an invariant effective radius is to be obtained.

In summary, we have used 18 different compounds, grouped into four isostructural families, to extract two equations that allow an estimation of the Radial Effective Charge model parameters. These equations not only give satisfactory parameters for the halide complexes presented in this work, they have already been used in two challenging systems where the lanthanoid ion is coordinated by oxygen ${ }^{25}$ and nitrogen $^{26}$ atoms. Thus, they are expected to be a very useful tool to address the widespread need for an inexpensive estimation of a starting parameter set in more sophisticated CF determinations, as well as a route for inexpensive qualitative prediction of the magnetic properties of f-block compounds.

\section{Acknowledgements}

The present work has been funded by the EU (ERC Advanced Grant SPINMOL and ERC Consolidator Grant DECRESIM), the Spanish MINECO (grant MAT2011-22785, MAT2014-56143-R, CTQ2014-52758-P), and the Generalitat Valenciana (Prometeo and ISIC Programmes of excellence). A.G.-A. acknowledges funding by the MINECO (Ramón y Cajal contract). J.J.B. thanks the Spanish MINECO for an FPU predoctoral grant.

\section{Notes and references}

1 J.-C. G. Bünzli, Acc. Chem. Res., 2006, 39(1), 53-61.

2 D. J. Newman, Adv. Phys., 1971, 20(84), 197-256.

3 C. A. Morrison and R. P. Leavitt, Spectroscopic properties of triply ionized, in Handbook on the physics and chemistry of rare earths, 1982, vol. 5, pp. 461-692.

4 C.-K. Duan and P. A. Tanner, J. Phys. Chem. A, 2010, 114, 6055-6062.

5 K. A. Schoene, J. R. Quagliano and F. S. Richardson, Inorg. Chem., 1991, 30, 3803-3812.

6 W. T. Carnall, P. R. Fields and B. G. Wybourne, J. Chem. Phys., 1965, 42, 3797.

7 R. Marx, F. Moro, M. Dörfel, L. Ungur, M. Waters, S. D. Jiang, M. Orlita, J. Taylor, W. Frey, L. F. Chibotaru and J. van Slageren, Chem. Sci., 2014, 5, 3287-3293.

8 K. S. Pedersen, L. Ungur, M. Sigrist, A. Sundt, M. SchauMagnussen, V. Vieru, H. Mutka, S. Rols, H. Weihe, O. Waldmann, L. F. Chibotaru, J. Bendix and J. Dreiser, Chem. Sci., 2014, 5, 1650-1660.

9 J. van Leusen, M. Speldrich, H. Schilder and P. Kögerler, Coord. Chem. Rev., 2014, 289-290, 137-148.

10 L. F. Chibotaru and L. Ungur, J. Chem. Phys., 2012, 137.

11 H. Bethe, Ann. Phys., 1929, 3, 133-208.

12 P. Porcher, M. C. Dos Santos and O. Malta, Phys. Chem. Chem. Phys., 1999, 1, 397-405.

13 W. Urland, Chem. Phys., 1976, 14, 393-401.

14 C. K. Jorgensen, R. Pappalardo and H. H. Schmidtke, J. Chem. Phys., 1963, 39, 1422-1430. 
15 O. L. Malta, Chem. Phys. Lett., 1982, 87, 27-29.

16 O. L. Malta, Chem. Phys. Lett., 1982, 88, 353-356.

17 Z. Zolnierek, J. Phys. Chem. Solids, 1984, 45(5), 523-528.

18 J. J. Baldoví, J. J. Borrás-Almenar, J. M. Clemente-Juan, E. Coronado and A. Gaita-Ariño, Dalton Trans., 2012, 41, 13705-13710.

19 M. A. Hughes, M. A. Lourenço, J. D. Carey, B. Murdin and K. P. Homewood, Opt. Express, 2014, 22(24), 29292-29303.

20 (a) J. J. Baldoví, S. Cardona-Serra, J. M. Clemente-Juan, E. Coronado and A. Gaita-Ariño, Chem. Sci., 2013, 4(3), 938-946; (b) J. J. Baldoví, J. M. Clemente-Juan, E. Coronado and A. Gaita-Ariño, Polyhedron, 2013, 66, 39-42; (c) J. J. Baldoví, E. Coronado, A. Gaita-Ariño, C. Gamer, M. Giménez-Marqués and G. Mínguez Espallargas, Chem. Eur. J., 2014, 20(34), 10695-10702; (d) B. Monteiro, J. T. Coutinho, C. C. L. Pereira, L. C. J. Pereira, J. Marçalo, M. Almeida, J. J. Baldoví, E. Coronado and A. Gaita-Ariño, Inorg. Chem., 2015, 54(4), 1949-1957; (e) K. Qian, J. J. Baldoví, S.-D. Jiang, A. Gaita-Ariño, Y.-Q. Zhang, J. Overgaard, B.-W. Wang, E. Coronado and S. Gao, Chem. Sci., 2015, DOI: 10.1039/C5SC01245G.

21 J. J. Baldoví, J. M. Clemente-Juan, E. Coronado, Y. Duan, A. Gaita-Ariño and C. Giménez-Saiz, Inorg. Chem., 2014, 53(18), 9976-9980.
22 (a) C. K. Jayasankar, F. S. Richardson and M. F. Reid, J. Less-Common Met., 1989, 148, 289-296; (b) C. K. Jayasankar, M. F. Reid and F. S. Richardson, Phys. Status Solidi, 1989, 155, 559; (c) G. K. Liu, W. T. Carnall, R. P. Jones, R. L. Cone and J. Huang, J. Alloys Compd., 1994, 207/208, 69-73; (d) P. A. Tanner, V. V. Ravi Kanth Kumar, C. K. Jayasankar and M. F. Reid, J. Alloys Compd., 1994, 215, 349-370; (e) P. A. Tanner, M. D. Faucher and X. Zhou, J. Phys. Chem. A, 2011, 115, 2557-2567; (f) X. Zhou, P. A. Tanner and M. D. Faucher, J. Phys. Chem. C, 2007, 111, 683-687; $(g)$ P. A. Tanner and M. D. Faucher, Chem. Phys. Lett., 2007, 445, 183-187.

23 (a) J. J. Baldovi, S. Cardona-Serra, J. M. Clemente-Juan, E. Coronado, A. Gaita-Ariño and A. Palii, J. Comput. Chem., 2013, 34, 1961-1967; (b) J. J. Baldovi, J. M. Clemente-Juan, E. Coronado, A. Gaita-Ariño and A. Palii, J. Comput. Chem., 2014, 35(26), 1930-1934.

24 P. A. Tanner and L. Ning, J. Phys. Chem. A, 2013, 117, 15031507.

25 J. J. Baldoví, Y. Duan, R. Morales, A. Gaita-Ariño, E. Ruiz and E. Coronado, submitted.

26 M. A. Antunes, J. T. Coutinho, I. C. Santos, J. Marçalo, L. C. J. Pereira, M. Almeida, J. J. Baldoví, A. Gaita-Ariño and E. Coronado, submitted. 